\title{
On Macdonald's formula for the volume of a compact Lie group
}

Yoshitake Hashimoto

Abstract. We give a simple proof of Macdonald's formula for the volume of a compact Lie group.

Mathematics Subject Classification (1991). 22E15.

Keywords. Compact Lie group, Todd class.

It is well-known that a compact Lie group $G$ of rank $r$ is rational homotopy equivalent to a product of spheres

$$
S=S^{2 m_{1}+1} \times \cdots \times S^{2 m_{r}+1}
$$

where $\left(m_{1}, \ldots, m_{r}\right)$ is called the exponent of $G$. In [3] I. G. Macdonald calculated the volume of $G$ with respect to the Haar measure $\mu$ induced from a Lebesgue measure $\lambda$ on the Lie algebra $\mathfrak{g}$ of $G$. The result is as follows:

$$
\mu(G)=\lambda\left(\mathfrak{g} / \mathfrak{g}_{\mathbb{Z}}\right) \sigma(S)
$$

where $\mathfrak{g}_{\mathbb{Z}}$ is the Chevalley lattice and $\sigma$ is the product of the superficial measure of the unit spheres $S^{2 m_{i}+1}$ in $\mathbb{R}^{2 m_{i}+2}$, that is

$$
\sigma\left(S^{2 m+1}\right)=\frac{2 \pi^{m+1}}{m !} .
$$

In this note we shall give a simpler proof of this formula.

Let $T$ be a maximal subgroup of $G, \mathfrak{t}$ its Lie algebra and $\mathfrak{t}_{\mathbb{Z}}$ a lattice in $\mathfrak{t}$ such that the kernel of the exponential map from $\mathfrak{t}$ to $T$ is $2 \pi \mathfrak{t}_{\mathbb{Z}}$. We fix a positive definite inner product on $\mathfrak{g}$ which is invariant under the adjoint action of $G$ such that the induced volume form coincides with $\lambda$. This inner product induces Lebesgue measures on $\mathfrak{t}$ and $\mathfrak{g} / \mathfrak{t}$ (also denoted by $\lambda$ ), which determine a Haar measure on $T$ and a $G$-invariant measure on the flag manifold $G / T$ (also denoted by $\mu$ ). Then clearly

$$
\mu(T)=(2 \pi)^{r} \lambda\left(\mathfrak{t} / \mathfrak{t}_{\mathbb{Z}}\right), \quad \mu(G)=\mu(T) \mu(G / T) .
$$


The main problem is to compute the volume of the flag manifold. We compute at a point of the flag manifold the ratio of the volume form $\mu$ and another $G$ invariant top form, the top term of the Todd class, whose integral value is known to be 1 [1]. The adjoint action of $G$ on $\mathfrak{g}$ induces trivialization of the direct sum of the tangent bundle of $G / T$ and a trivial $\mathfrak{t}$ bundle. Hence the rational Pontrjagin classes of $G / T$ all vanish and the Todd class equals $\exp \frac{1}{2} c_{1}(G / T)$. The top term is $\frac{1}{n !}\left(\frac{1}{2} c_{1}\right)^{n}$ where $n$ is the complex dimension of $G / T$. Let $X_{\alpha} \in \mathfrak{g} \otimes \mathbb{C}$ be an eigenvector for a root $\alpha$ such that $\frac{1}{2}\left[X_{\alpha}, X_{-\alpha}\right]=\alpha^{\vee}$, the coroot for $\alpha$, then

$$
\frac{1}{2} c_{1}\left(X_{\alpha}, X_{\beta}\right)= \begin{cases}\frac{\left\langle\rho \mid \alpha^{\vee}\right\rangle}{\pi}, & \beta=-\alpha, \\ 0, & \beta \neq-\alpha .\end{cases}
$$

where

$$
\rho=\frac{1}{2} \sum_{\alpha>0} \alpha
$$

on the other hand

$$
\left\langle X_{\alpha}, X_{-\alpha}\right\rangle=\left|\alpha^{\vee}\right|^{2} .
$$

Evaluating the volume form and $\frac{1}{n !}\left(\frac{1}{2} c_{1}\right)^{n}$ for $2 n$ vectors $\left(X_{\alpha}, X_{-\alpha} ; \alpha>0\right)$, we obtain

$$
\prod_{\alpha>0}\left|\alpha^{\vee}\right|^{2}, \quad \prod_{\alpha>0} \frac{\left\langle\rho \mid \alpha^{\vee}\right\rangle}{\pi}
$$

respectively. Since $\alpha^{\vee}$ is the sum of $\left\langle\rho \mid \alpha^{\vee}\right\rangle$ simple coroots [2], it holds that

$$
\prod_{\alpha>0}\left\langle\rho \mid \alpha^{\vee}\right\rangle=\prod_{i=1}^{r}\left(m_{i} !\right) .
$$

Hence

$$
\mu(G / T)=\frac{\sigma(S)}{(2 \pi)^{r}} \prod_{\alpha>0}\left|\alpha^{\vee}\right|^{2} .
$$

The final result follows from

$$
\lambda\left(\mathfrak{g} / \mathfrak{g}_{\mathbb{Z}}\right)=\lambda\left(\mathfrak{t} / \mathfrak{t}_{\mathbb{Z}}\right) \prod_{\alpha>0}\left|\alpha^{\vee}\right|^{2} .
$$

The author would like to thank K. Abe, A. Hattori and K. Ono for useful comments and encouragement. 


\section{References}

[1] A. Borel and F. Hirzebruch, Characteristic classes and homogeneous spaces II, Amer. J. Math. 81 (1959), 315-382.

[2] N. Bourbaki, Groupes et algèbres de Lie, chapitres 4, 5 et 6, Hermann, Paris 1968.

[3] I. G. Macdonald, The volume of a compact Lie group, Invent. math. 56 (1980), 93-95.

Yoshitake Hashimoto

Department of Mathematics

Osaka City University

Osaka, Japan

(Received: May 10, 1997) 